\title{
A new approach to hydraulic calculations of free-flow drainage systems
}

\author{
Elena Kitaytseva ${ }^{1^{*}}$ \\ ${ }^{1}$ Moscow state university of civil engineering, Yaroslavskoye shosse, 26, Moscow, Russia, 129337
}

\begin{abstract}
The accuracy of hydraulic calculations is determined by the accuracy of the mathematical models inherent in their foundation. The results of calculations, obtained by different models, and differing from each other by $5-10 \%$ is considered identical. The present study examines classical ways of finding the Chezy formula that are used for calculations of free-flow pipelines. These formulas differ from each other not only in parameters but also in the form of the functional dependence. The equation, underlying the hydraulic calculations, is nonlinear. Its solution is possible only in the number of well-established methods. It offers numerical computing to find pipelines diameters and their filling, as well as the algorithm to realize it. It conducts numerical experiment to compare pipelines diameters and filling and flow velocities. It presents frequency characteristics to distribute the parameters under analysis. For a specific example, the difference of cost of pipes, the diameters of which was found using the various formulas for coefficient Chezy, was calculated.
\end{abstract}

\section{Introduction}

According to [1] "hydraulic calculation of sewerage free-flow pipelines should be carried out using charts, diagrams and nomograms". The A.A. Lukinykh and N.A. Lukinykh charts [2] that are widely accepted in engineering practice for calculations of free-flow drainage systems are based on N. N. Pavlovsky's function [3] and allow to do these tasks. There are online calculators automatizing use of these charts. When working out programs, the "hand counting" algorithm is used most frequently where the solution is sought by exhaustion [4].

\section{Literature review}

Hydraulic calculations of free-flow (gravity) pipelines are made if water flow should be stable and steady. These calculations are based on the assumption about the continuity of the flow:

$$
q=1000 \omega v
$$

and the Chezy formula:

$$
v=C \cdot \sqrt{R \cdot i}
$$

\footnotetext{
*Corresponding author: keh2@bk.ru
} 
where $\mathrm{q}$ is flow rate, $1 / \mathrm{s} ; \omega$ is water area, $\mathrm{m}^{2} ; v$ is flow velocity, $\mathrm{m} / \mathrm{s} ; \mathrm{R}$ is hydraulic radius, $\mathrm{m}$; $\mathrm{i}$ is hydraulic slope; $\mathrm{C}$ is the Chezy coefficient, depending on hydraulic radius and roughness of wetted surface of pipelines, $\mathrm{m}^{0,5} / \mathrm{s}$.

When the pipe is filled partially the hydraulic radius $R$, water area $\omega$ and filling $h / D$ can be stated in term of solid angle $\phi$ :

$$
\begin{aligned}
R & =\frac{D}{4}\left(1-\frac{\sin \varphi}{\varphi}\right) \\
\omega & =\frac{D^{2}}{8}(\varphi-\sin \varphi) \\
\frac{h}{D} & =\frac{1}{2}\left[1+\sin \frac{180-\varphi}{2}\right]
\end{aligned}
$$

where $\mathrm{D}, \mathrm{m}$, is inner pipe diameter.

The fundamental difficulty in making hydraulic calculations is to find the Chezy coefficient $C$. The N. N. Pavlovsky formula prevails:

$$
\begin{aligned}
& C=\frac{R^{y}}{n_{1}} \\
& y=2.5 \sqrt{n_{1}}-0.13-0.75 \sqrt{R}\left(\sqrt{n_{1}}-0.1\right)
\end{aligned}
$$

where $n_{1}$ is relative roughness of pipe surface.

Alongside with this one, formulas developed by other authors are used, e.g.:

The S.V. Yakovlev formula:

$$
C=24.7 \cdot \lg \left(\frac{10^{3} R v}{v \varepsilon+0.01}\right)
$$

where $\varepsilon, \mathrm{mm}$, is absolute surface roughness;

The A. D. Altshul formula:

$$
C=20\left(\frac{1000 R}{k_{e}+\frac{0.004}{\sqrt{1000 R i}}}\right)^{\frac{1}{6}}
$$

where $\mathrm{k}_{\mathrm{e}}, \mathrm{mm}$ is equivalent steady grained roughness;

The N. F. Fedorov formula:

$$
C=\sqrt{\frac{8 g}{\lambda}}
$$

where $\lambda$ is hydraulic friction coefficient that can be found with the help of the formula:

$$
\frac{1}{\sqrt{\lambda}}=-2 \lg \left(\frac{\Delta_{e}}{13680 R}+\frac{a_{2}}{\operatorname{Re}}\right)
$$

$R e$ is the Reynolds number

$$
\operatorname{Re}=10^{4} \frac{8 q}{v \varphi D}
$$

$\Delta_{\mathrm{e}}, \mathrm{mm}$, is equivalent absolute roughness; $\mathrm{a}_{2}$, is the coefficient which takes into account the characteristics of pipe surface roughness; $v, \mathrm{~m}^{2} / \mathrm{s}$, is kinematic viscosity.

The coefficients from dependences $(6-9,11)$, characterize inner surface roughness in their own way. If the pipe material is given, they are defined with help of reference data [5]. For new materials the roughness values are found experimentally $[6,7]$. In the research [8] the relation between coefficients $\mathrm{n}_{1}$ and $\mathrm{k}_{\mathrm{e}}$ is deduced. 
Prescriptive literature [1] recommends using the N. N. Pavlovsky formula (6-7) and the N. F. Fedorov formula (10-12); in the meanwhile, it is underlined in [9] that formulas (6-7) are "right for areas of quite rough rubbing". At the same time a number of authors $[10,11]$ point to the opportunity for pipelines to work in the transition area where the Chezy formula depends on velocity (8) or the Reynolds number (11).

The formulas (6-12) are empirical or semiempirical dependencies and according to the authors, they correspond with experimental data well.

\section{Methods and materials}

Combining dependencies $(1,2)$ we get equation:

$$
f(D, i, \varphi)=q-1000 \cdot \omega \cdot C \cdot \sqrt{R \cdot i}=0
$$

When the flow rate is known, the equation (13) can be used to solve the following problems:

- to find diameter if pipe filling and slope are known;

- to find filling if pipe diameter and slope are known;

- to find slope if pipe diameter and filling are known.

The offered algorithm consists of the following steps:

1. To set the initial approximation to solid angle $\phi^{(0)}=3.55$;

2. To set the initial approximation to slope $\mathrm{i}^{(0)}$ : for off-nominal sectors $-\mathrm{i}^{(0)}=0.007$, for nominal sectors $\mathrm{i}^{(0)}=\min \left(0.00956 \mathrm{q}^{0.306} ; 0.007\right)$;

3. To assign $\mathrm{i}^{(\mathrm{k})}=\mathrm{i}^{(0)}$;

4. To set initial approximation to $\mathrm{D}^{(0)}=160$ (minimal permissible diameter for outer drainage systems);

5. To assign $\mathrm{D}^{(\mathrm{k})}=\mathrm{D}^{(0)}$;

6. To solve equation (13) for the unknown D;

7. To take as the answer a bigger diameter $\mathrm{D}$ which is nearest in pipe grade depending on pipe material;

8. To specify more exactly slope $\mathrm{i}^{(\mathrm{k}+1)}=\max \left(\mathrm{i}^{(\mathrm{k})} ; 1 / \mathrm{D}\right)$;

9. To specify initial approximation to solid angle $\phi^{(0)}$ according to the determined value of diameter D using the recommendations [2];

10. To assign $\phi^{(\mathrm{k})}=\phi^{(0)}$;

11. To solve equation (13) for the unknown $\phi$;

12. To compute the filling $\mathrm{h} / \mathrm{d}$ using the formula (5) and to compute the velocity $\mathrm{v}$ using the formula (2).

It is possible to solve the equation (13), which is nonlinear for the unknown $\mathrm{D}$, only by means of numerical method $[12,13]$. The Newton method was chosen as numerical method:

$$
D^{(k+1)}=D^{(k)}-\frac{f\left(D^{(k)}, i, \varphi\right)}{f^{\prime}\left(D^{(k)}, i, \varphi\right)}
$$

where $\mathrm{k}=0,1,2, \ldots$ is iteration number;

$$
\begin{aligned}
f^{\prime}\left(D^{(k)}, i, \varphi\right) & =\frac{\partial f}{\partial D}=1000 \sqrt{i}\left[\frac{\partial \omega}{\partial D} \cdot C \cdot \sqrt{R}+\omega\left(\frac{\partial C}{\partial D} \sqrt{R}+\frac{C}{2 \sqrt{R}} \frac{\partial R}{\partial D}\right)\right] \\
\frac{\partial \omega}{\partial D} & =\frac{D}{4}(\varphi-\sin \varphi) \\
\frac{\partial R}{\partial D} & =\frac{1}{4}\left(1-\frac{\sin \varphi}{\varphi}\right)
\end{aligned}
$$


Iterations were over on the condition that:

$$
\left|f\left(D^{(k)}, i, \varphi\right)\right|<0.001
$$

According to the model used, the derivative of the Chezy formula $\frac{\partial C}{\partial D}$ is calculated using the following formulas: The N. N. Pavlovsky model

$$
\frac{\partial C}{\partial D}=C\left[\frac{\partial y}{\partial D} \cdot \ln R+\frac{y}{R} \cdot \frac{\partial R}{\partial D}\right],
$$

where

$$
\frac{\partial y}{\partial D}=-\frac{0.375\left(\sqrt{n_{1}}-0.1\right)}{\sqrt{R}} \frac{\partial R}{\partial D}
$$

- the S. V. Yakovlev model

$$
\frac{\partial C}{\partial D}=\frac{24.7}{\ln 10}\left[\frac{1}{R} \cdot \frac{\partial R}{\partial D}+\left(\frac{1}{v}-\frac{\varepsilon}{v \varepsilon+0.01}\right) \frac{\partial v}{\partial D}\right]
$$

where

$$
\frac{\partial v}{\partial D}=-\frac{q}{1000 \omega^{2}} \frac{\partial \omega}{\partial D}
$$

- the A. D. Altshul model

$$
\frac{\partial C}{\partial D}=\frac{20}{6}\left(\frac{1000 \cdot R}{k_{e}+\frac{0.004}{\sqrt{1000 R i}}}\right)^{-\frac{5}{6}} \cdot \frac{1000 \cdot\left(k_{e}+\frac{0.004}{\sqrt{1000 R i}}\right)+\frac{2 \sqrt{1000 i}}{\sqrt{R}}}{\left[k_{e}+\frac{0.004}{\sqrt{1000 R i}}\right]^{2}} \cdot \frac{\partial R}{\partial D}
$$

- the N. F. Fedorov model

$$
\frac{\partial C}{\partial D}=\frac{4 \sqrt{2 g}}{\ln 10} \cdot \frac{\frac{\Delta_{e}}{13.68 R^{2}} \cdot \frac{\partial R}{\partial D}+\frac{a_{2}}{\mathrm{Re}^{2}} \cdot \frac{\partial \mathrm{Re}}{\partial D}}{\frac{\Delta_{e}}{13.68 R}+\frac{a_{2}}{\operatorname{Re}}}
$$

where

$$
\frac{\partial \mathrm{Re}}{\partial D}=-10^{4} \frac{8 q}{v \varphi D^{2}}
$$

As in most cases, the calculated diameter differs from the standard one, which is taken as the answer, it is necessary to specify the filling of pipelines $h / D$ solving the equation (13) for the unknown solid angle $\phi$. As this method the Newton method is used:

$$
\varphi^{(k+1)}=\varphi^{(k)}-\frac{f\left(D, i, \varphi^{(k)}\right)}{f^{\prime}\left(D, i, \varphi^{(k)}\right)}
$$

where $\mathrm{k}=0,1,2, \ldots$ is iteration number;

$$
\begin{aligned}
& f^{\prime}\left(D, i, \varphi^{(k)}\right)=\frac{\partial f}{\partial \varphi}=1000 \cdot \sqrt{i}\left[\frac{\partial \omega}{\partial \varphi} \cdot C \cdot \sqrt{R}+\omega\left(\frac{\partial C}{\partial \varphi} \sqrt{R}+\frac{C}{2 \sqrt{R}} \frac{\partial R}{\partial \varphi}\right)\right] \\
& \frac{\partial \omega}{\partial \varphi}=\frac{D^{2}}{8}(1-\cos \varphi)
\end{aligned}
$$




$$
\frac{\partial R}{\partial \varphi}=-\frac{D}{4} \cdot \frac{\varphi \cdot \cos \varphi-\sin \varphi}{\varphi^{2}}
$$

Iterations are over on the condition that:

$$
\left|f\left(D, i, \varphi^{(k)}\right)\right|<0.001
$$

According to the model, which is used, the derivative of the Chezy formula $\frac{\partial C}{\partial \varphi}$ is calculated using the formulas:

- the N. N. Pavlovsky model

$$
\frac{\partial C}{\partial \varphi}=C\left[\frac{\partial y}{\partial \varphi} \cdot \ln R+\frac{y}{R} \cdot \frac{\partial R}{\partial \varphi}\right],
$$

where

$$
\frac{\partial y}{\partial \varphi}=-\frac{0.375\left(\sqrt{n_{1}}-1\right)}{\sqrt{R}} \frac{\partial R}{\partial \varphi}
$$

- the S. V. Yakovlev model

$$
\frac{\partial C}{\partial \varphi}=\frac{24.7}{\ln 10}\left[\frac{1}{R} \cdot \frac{\partial R}{\partial \varphi}+\left(\frac{1}{v}-\frac{\varepsilon}{v \varepsilon+0.01}\right) \frac{\partial v}{\partial \varphi}\right]
$$

where

$$
\frac{\partial v}{\partial \varphi}=-\frac{q}{\omega^{2}} \frac{\partial \omega}{\partial \varphi}
$$

- the A. D. Altshul model

$$
\frac{\partial C}{\partial \varphi}=\frac{20}{6}\left(\frac{1000 \cdot R}{k_{e}+\frac{0.004}{\sqrt{1000 R i}}}\right)^{-\frac{5}{6}} \cdot \frac{1000 \cdot\left(k_{e}+\frac{0.004}{\sqrt{1000 R i}}\right)+\frac{2 \sqrt{1000 i}}{\sqrt{R}}}{\left[k_{\ni}+\frac{0.004}{\sqrt{1000 R i}}\right]^{2}} \cdot \frac{\partial R}{\partial \varphi}
$$

- the N. F. Fedorov model

$$
\frac{\partial C}{\partial \varphi}=\frac{4 \sqrt{2 g}}{\ln 10} \cdot \frac{\frac{\Delta_{e}}{13.68 R^{2}} \cdot \frac{\partial R}{\partial \varphi}+\frac{a_{2}}{\operatorname{Re}^{2}} \cdot \frac{\partial \mathrm{Re}}{\partial \varphi}}{\frac{\Delta_{e}}{13.68 R}+\frac{a_{2}}{\operatorname{Re}}}
$$

where

$$
\frac{\partial \operatorname{Re}}{\partial \varphi}=-10^{4} \frac{8 q}{v \varphi^{2} D}
$$

The developed algorithm was realized for Excel Microsoft Office 2007.

\section{Research}

The numerical experiment was carried out to compare the results which were received in numerical way using the N. N. Pavlovsky (P) model (6-7), the S. V. Yakovlev (Ya) model (8), the A. D. Altshul (A) model (9), the N. F. Fedorov (F) model (10-11) for the Chezy formula.

The scheme of the drainage system presented in Fig. 1 was used as research object. The scheme contained 126 pipelines, 35 of which are off-nominal $(q<10)$. The flow rates in the 
pipelines varied within the limits $1.9-278.1$ 1/s. The lengths of the pipelines varied from 8 to $1615 \mathrm{~m}$, the overall length of the whole system was $45.506 \mathrm{~km}$.

The above algorithm was used to find the pipeline diameter and its filling for all the pipelines. With the assigned accuracy of $0.001 \mathrm{l} / \mathrm{s}$ the number of iterations in iteration processes (14) and (27) did not exceed 8.

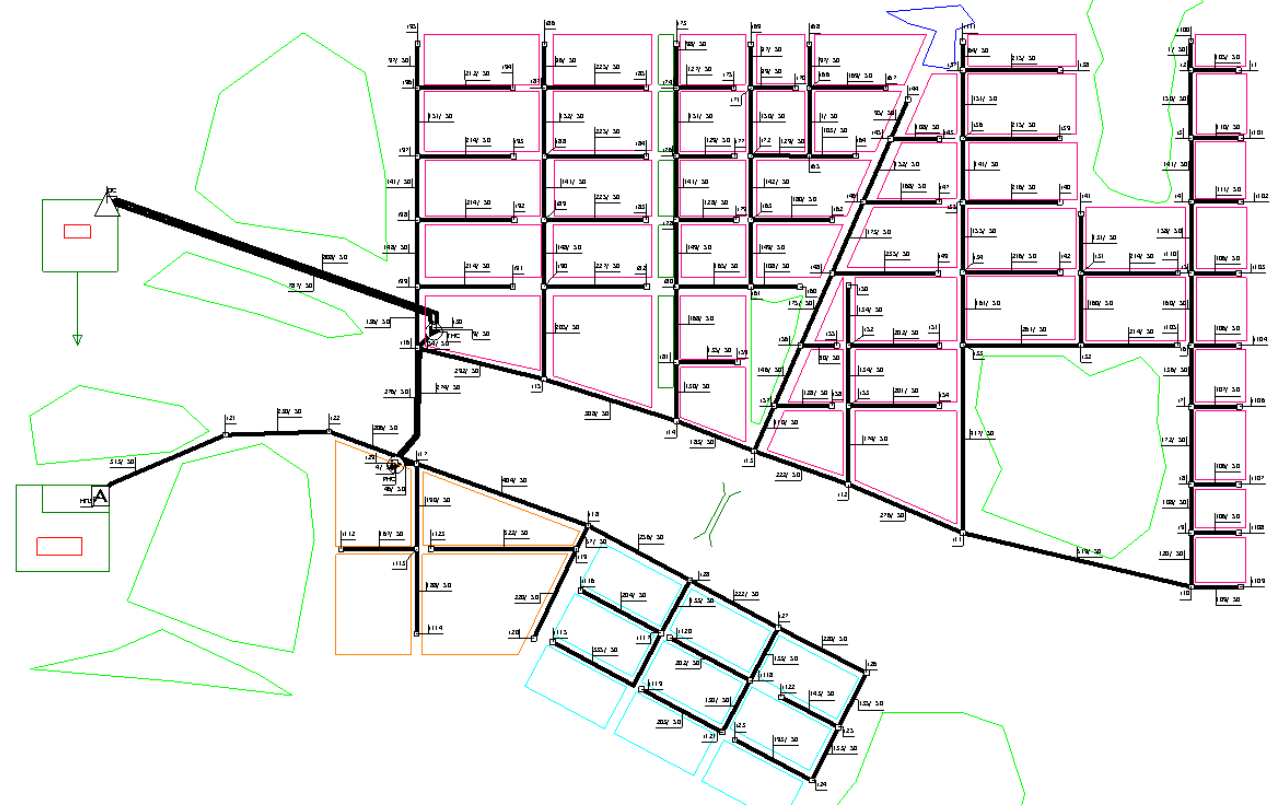

Fig. 1. Drainage System Scheme

Table 1. Combined parameters of comparison of hydraulic calculations results

\begin{tabular}{|c|c|c|c|c|c|c|c|c|c|c|}
\hline \multirow{4}{*}{$\begin{array}{l}\text { Set of } \\
\text { pipelines }\end{array}$} & & \multicolumn{9}{|c|}{ Parameter } \\
\hline & & \multicolumn{3}{|c|}{$\left(\mathrm{D}_{\text {in }}-\mathrm{D}_{\text {in }}{ }^{(\mathrm{P})}\right) / \mathrm{D}_{\text {in }}{ }^{(\mathrm{P})} * 100, \%$} & \multicolumn{3}{|c|}{$\left(\mathrm{h} / \mathrm{d}-\mathrm{h} / \mathrm{d}^{(\mathrm{P})}\right) / \mathrm{h} / \mathrm{d}^{(\mathrm{P}) * 100, \%}$} & \multicolumn{3}{|c|}{$\left(\mathrm{v}-\mathrm{v}^{(\mathrm{P})}\right) / \mathrm{v}^{(\mathrm{P}) *} 100, \%$} \\
\hline & & \multicolumn{9}{|c|}{ Model } \\
\hline & & $\mathrm{Ya}$ & A & F & $\mathrm{Ya}$ & A & $\mathrm{F}$ & $\mathrm{Ya}$ & A & $\mathrm{F}$ \\
\hline \multirow{4}{*}{ 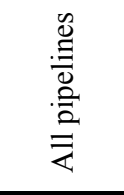 } & $\min$ & -1.36 & -4.75 & -7.20 & -2.17 & -7.79 & -11.53 & -14.31 & 5.48 & 6.63 \\
\hline & $\max$ & 8.24 & -2.81 & -3.55 & 11.54 & -3.69 & -4.42 & 2.80 & 10.41 & 16.27 \\
\hline & $\mathrm{m}$ & 4.05 & -3.37 & -5.25 & 6.39 & -5.25 & -7.97 & -7.49 & 7.12 & 11.21 \\
\hline & $\mathrm{s}$ & 1.97 & 0.45 & 0.72 & 3.25 & 0.85 & 1.26 & 3.89 & 1.13 & 1.80 \\
\hline \multirow{4}{*}{ 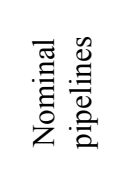 } & $\min$ & -1.36 & -4.75 & -7.20 & -2.17 & -7.79 & -11.53 & -8.72 & 6.65 & 10.12 \\
\hline & $\max$ & 4.70 & -3.18 & -4.72 & 7.56 & -4.89 & -7.22 & 2.80 & 10.41 & 16.27 \\
\hline & $\mathrm{m}$ & 3.16 & -3.51 & -5.40 & 4.85 & -5.63 & -8.43 & -5.64 & 7.56 & 11.76 \\
\hline & $\mathrm{s}$ & 1.76 & 0.48 & 0.79 & 3.11 & 0.80 & 1.18 & 3.69 & 1.19 & 1.88 \\
\hline
\end{tabular}

\section{Results}

The pipelines diameters $D_{\text {in }}$, their filling $\mathrm{h} / \mathrm{d}$, the flow velocity $\mathrm{v}$ were studied as analyzed parameters. For every pipeline the values $D_{i n}, h / d, v$, received with the using of the $S$. V. 
Yakovlev, A. D. Altshul, N. F. Fedorov models, were compared with the respective values $\mathrm{D}_{\text {in }}{ }^{(\mathrm{P})}, \mathrm{h} / \mathrm{d}^{(\mathrm{P})}, \mathrm{v}^{(\mathrm{P})}$ received with the using of the N. N. Pavlovsky model.

Two sets were analyzed (chart 1): the first set included all the pipelines, the second one comprised only nominal pipelines for which $\mathrm{q}>10 \mathrm{l} / \mathrm{s}$. The filling $\mathrm{h} / \mathrm{d}$ and velocity $\mathrm{v}$ were compared only for those pipelines where $\mathrm{D}_{\text {in }}$ coincided with $\mathrm{D}_{\mathrm{in}}{ }^{(\Pi)}$. For every sample and each parameter, the minimum, maximum, average values and standard divergence were evaluated. The removal of off-nominal sectors from sample 1 does not change minimum and average values of the studied parameters considerably, at the same time it decreases peak-to-peak value/ amplitude and standard divergence.

\section{Discussion}

The analysis that was conducted showed that the diameters which were calculated with the help of the S. V. Yakovlev model are by $3 \%$ in average bigger than the diameters calculated with the help of the N. N. Pavlovsky model whereas the diameters calculated with the help of the A. D. Altshul and the N. F. Fedorov models are by $3.5 \%$ and $5.4 \%$ respectively smaller than the diameters calculated using the N. N. Pavlovsky model (Fig. 2). The filling of the pipelines that was calculated using the S. V. Yakovlev model, is by $4.85 \%$ in average bigger than the filling calculated using the N.N. Pavlovsky model whereas the filling calculated using the A. D. Altshul and the N. F. Fedorov models is by $5.63 \%$ and $8.43 \%$ respectively smaller than the filling calculated with the help of the N. N. Pavlovsky model (Fig. 3). The flow velocity that was calculated with the help of the S. V. Yakovlev model, is by $5.64 \%$ in average smaller than the velocity calculated using the N. N. Pavlovsky model whereas the velocity calculated using the A. D. Altshul and N. F. Fedorov models is by $7.56 \%$ and $11.76 \%$ respectively bigger than the velocity calculated using the N. N. Pavlovsky model (Fig. 4).

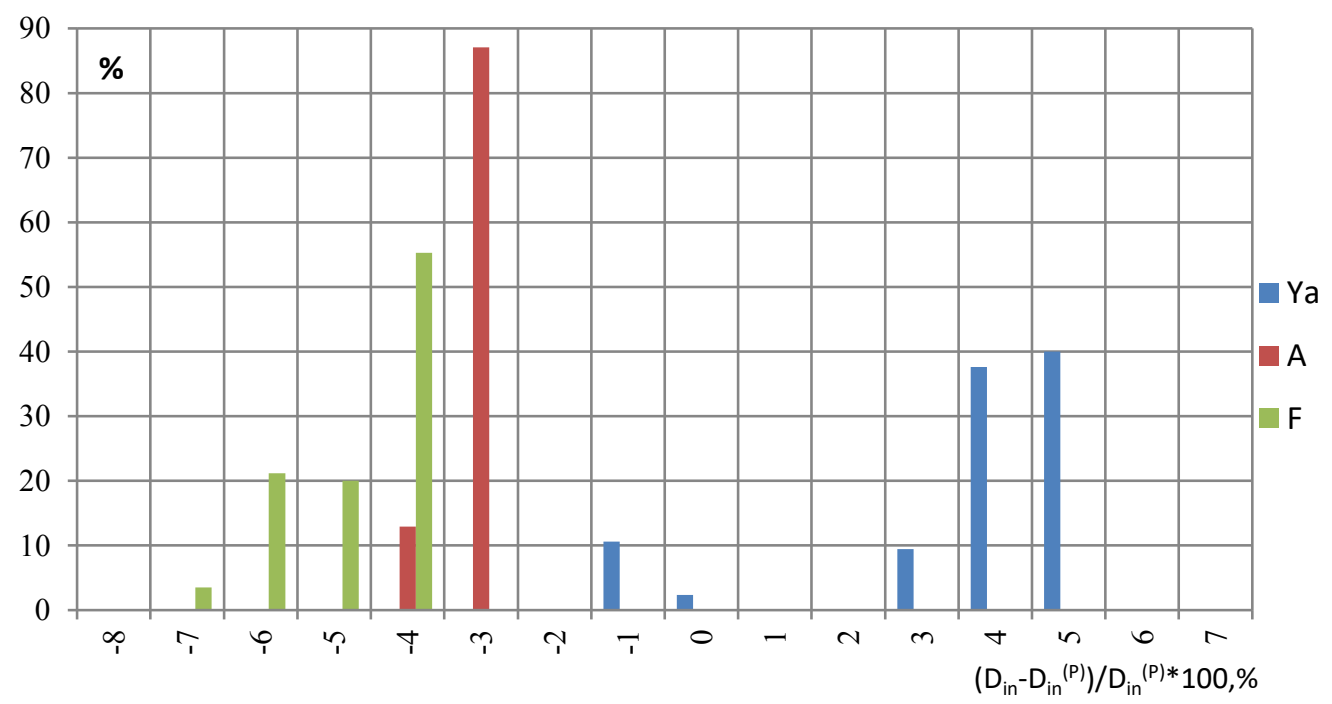

Fig. 2. Frequency characteristics of distribution of inner diameters calculated by different models 


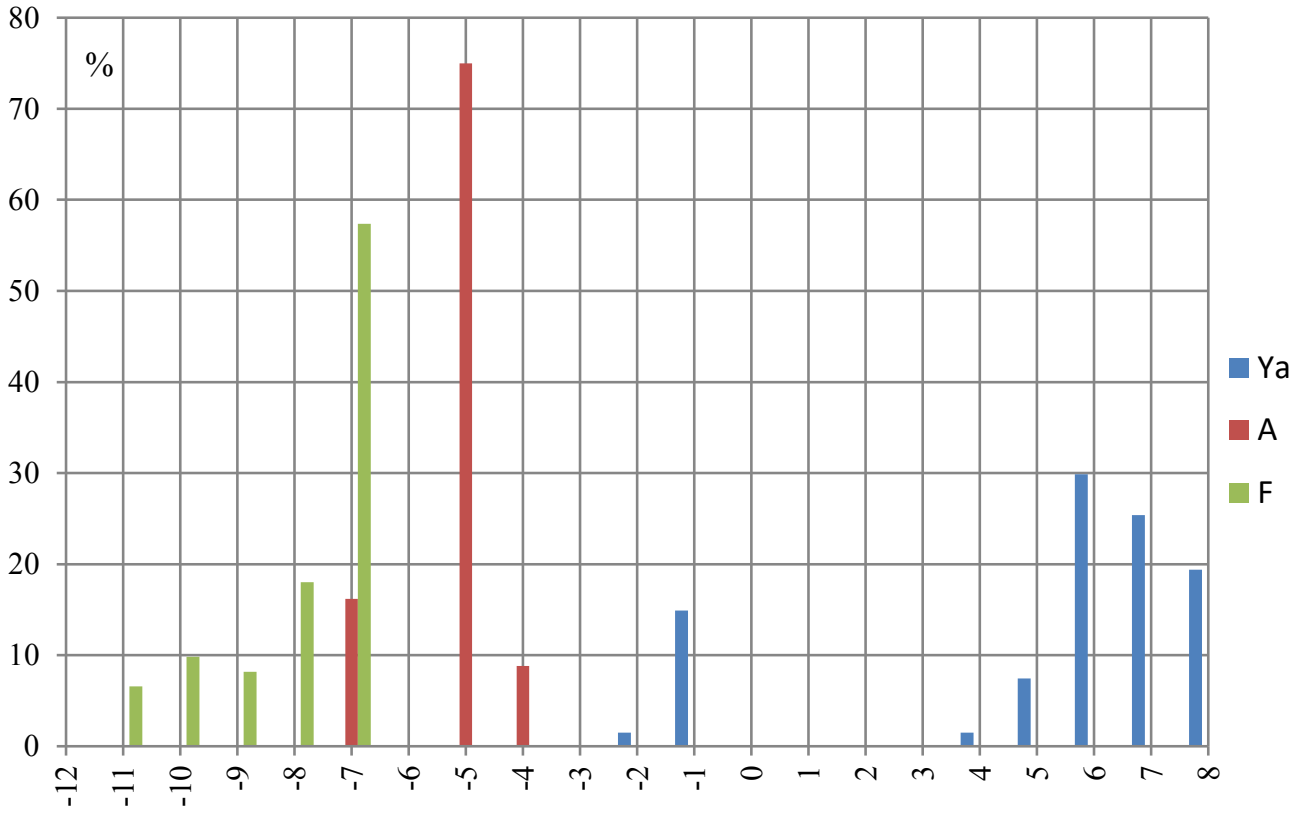

$\left(\mathrm{h} / \mathrm{d}-\mathrm{h} / \mathrm{d}^{(\mathrm{P})}\right) / \mathrm{h} / \mathrm{d}^{(\mathrm{P}) *} 100, \%$

Fig. 3. Frequency characteristics of distribution of pipes filling calculated by different models

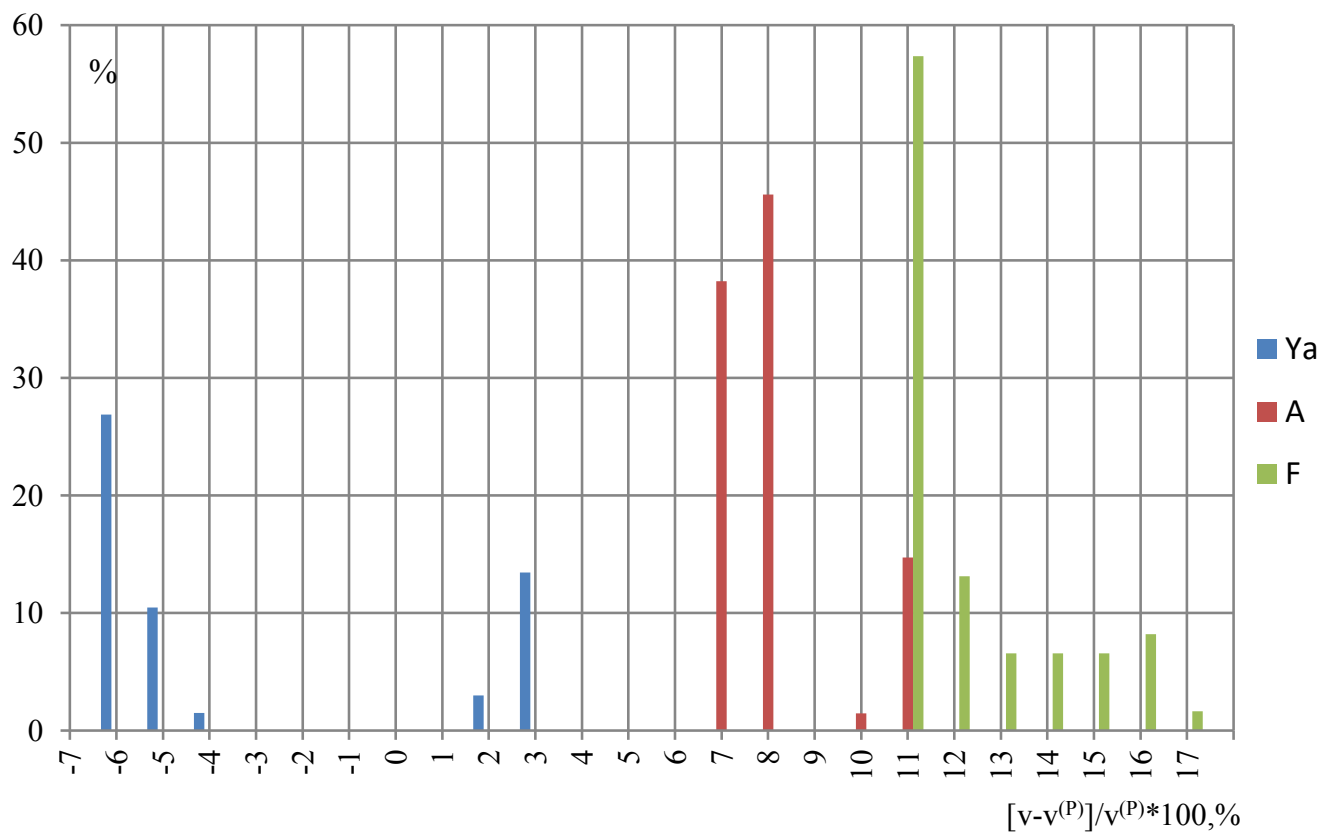

Fig. 4. Frequency characteristics of distribution of velocity calculated by different models

From the viewpoint of the accuracy of the results that were received, all the four models are acceptable for engineering calculations. However, matching the cost of pipes for the overall drainage system makes a stronger impression. The difference in diameters on the one hand allows saving 6.924 or 4.783 million roubles by means of using the N. F. Fedorov and 
the A. D. Altshul models respectively, on the other hand it increases expenses by 4.195 million roubles in case of using the S. V. Yakovlev model.

\section{Conclusions}

1. The recommended use of charts, diagrams and nomograms for hydraulic calculations does not satisfy the contemporary level of Information Technologies.

2. Numerical methods should be used in hydraulic calculations. Iteration methods have stable and rapid convergence.

3. The adequacy of the N. N. Pavlovsky, the A. D. Altshul, the N. F. Fedorov and S. V. Yakovlev models to the physics process can be verified by laboratory or natural experiment. The N. N. Pavlovsky model has the longest history of utilization, so the results received by this model, were chosen as the base set.

4. The fillings of the pipes and the speed of the streams received in all models keep within the admissible limits.

5. The N. F. Fedorov model (11) taking into account an important parameter, namely the Reynolds number Re, allows in certain cases to fit smaller diameters. It consequently allows decreasing expenses when purchasing pipes.

\section{References}

1. Svod pravil SP 32.13330.2012 "SNiP 2.04.03-85. Kanalisatsiya. Naruzhniye sety i soorugeniya" Aktualizirovannaya redaktsiya SNiP 2.04.03-85. (2012)

2. A.A. Lukinikh, N.A. Lukinikh, Tablitsy dlya guidravlicheskogo rascheta kanalizatsyonnyh setey po formule akad. Pavlovskogo N.N. (1974)

3. P.G. Kiselev, Spravochnik po gidravlicheskim raschetam (1972)

4. A.U. Khemich, A.V. Atkin, Internet-vesnik VolgGASU, 2 (4) (2007)

5. A.M. Kurganov, N.F. Fedorov, Guidravlicheskiye raschety system vodosnabgeniya i vodootvedeniya: Spravochnik (1986)

6. V.A. Orlov, R.E. Khurgin, Vesnik MGSU, 3, 118-122 (2010)

7. Orlov V.A., Zotkin S.P, Orlov E.V., Khurgin R.E., Maleeva A.V., Vestnik MGSU, 3, 205-210 (2012)

8. Rylova I.A., Borovkov V.S., Vestnik MGSU, 4, 181-187 (2013)

9. Kalitsyn V.I. Guidravlicheskiye soprotivleniya samotechnikh vodootvodjashikh setej (1986)

10. Bachir Achour, The Open Civil Eng. J., 9, 187-195 (2015)

11. Kalitsyn V.I. Vodootvodjashiye systemy I soorugeniya (1987)

12. Janet Bacro, Kenneth M. Wong, Michael K. Stenstrom// J. of Hydr. Eng., 134(4), 466474 (2008)

13. Pushpa N. Rathie, Prabhata K. Swamee, Luan Carlos de S. M. Ozelim// Appl. Math. and Phys., 1 (4), 120-125 (2013) 